\title{
OBSERVATIONS OF QUASAR BROAD-LINE PROFILES IN THE OPTICAL AND INFRARED
}

\author{
Keith Louis Thompson \\ Astronomy Department, University of Texas at Austin \\ Austin, Texas 78712
}

Seven low redshift QSOs have been observed spectroscopically at infrared and optical wavelengths at McDonald Observatory. The two instruments used were the infrared grating spectrometer (IRGS; see Lester, Harvey, and Carr 1988 for a description) on the $2.7 \mathrm{~m}$ reflector, and the es- 2 cassegrain spectrometer on the $2.1 \mathrm{~m}$. Table 1 shows the objects and spectra taken.

The optical spectra include $\mathrm{H} \alpha$, $\mathrm{He}$ I $\lambda 5876$, and $\mathrm{H} \beta$, observed at $\sim 14 \AA$ resolution (FWHM). The targets for the infrared spectra were $\mathrm{Pa} \alpha$, He I $\lambda 10830$, and $\mathrm{Pa} \gamma . \mathrm{Pa} \alpha$ has been observed in most of the objects with a resolution $\lambda / \Delta \lambda \sim 200(\sim 0.0107 \mu \mathrm{m})$. Each spectrum consists of two integrations, with the grating shifted by half a channel so as to fully Nyquist sample the spectrum. Due to the design of the IRGS, this instrument is not optimum for observations of $\mathrm{He} \mathrm{I} \lambda 10830$ and Pay in the J-band; only the brightest objects are observable with sufficient signal to noise ratio and spectral resolution; they were not Nyquist sampled, but observed at a higher resolution. See figures 1, 2, and 3 for the spectra of $1226+023$.

The goal of this work is to compare the broad-line profile ratios with photoionization models to help determine the gas conditions as a function of velocity projected on our line of sight. From this we hope to put constraints on kinematic models. This approach has been used by several researchers in the past to study quasar and Seyfert 1 broad lines (e.g. Shuder 1982, 1984, Crenshaw 1986). However, the number of lines useful for this type of study is limited to two or three if taken from the optical region for low redshift quasars, and this is why we have turned to these near infrared lines. Theoretically, we need at least as many line ratios as there are parameters in our photoionization models just to determine the conditions of a single cloud.

A grid of photoionization models has been run with G. Ferland's code CLOUDY using the University of Texas Center for High Performance Computing CRAY XMP/24.

Because the continuum was chosen to match that of 1226+023 (see Perry, Ward, and Jones 1987), which has an unusual spectrum, more grids need to be made using continua appropriate to more typical QSOs. The results, however, suggest that the ratios $\mathrm{Pa} \alpha / \mathrm{H} \alpha$ and $\mathrm{He} \mathrm{I} \lambda 5876 / \lambda 10830$ are both useful in determining gas density, cloud thickness, ionization parameter, and reddening.

Crenshaw 1986, Ap. J. Suppl., 62, 821. 
Lester, Harvey, and Carr 1988, Ap. J., 329, 641.

Perry, Ward, and Jones 1987, Mon. Not. R. astr. Soc., 228, 623.

Shuder 1982, Ap. J., 259, 48.

Shuder 1984, Ap. J., $280,491$.

Table 1. objects observed during this project

$\begin{array}{lccccc}\text { name } & \mathbf{z} & \text { spectra: } & \text { optical } & \text { Pa } \alpha & \text { He I } \lambda 10830 \\ 0026+129 & 0.142 & & \text { x } & -- & -- \\ 0804+761 & 0.100 & \text { x } & \text { x } & -- \\ 1211+14 & 0.085 & \text { x } & \text { x } & -- \\ 1226+023 & 0.158 & \text { x } & \text { x } & \text { x } \\ 13349+2438 & 0.108 & \text { x } & \text { x } & \text { x } \\ 1612+26 & 0.131 & \text { x } & \text { x } & -- \\ 1803+676 & 0.136 & \text { x } & \text { x } & --\end{array}$

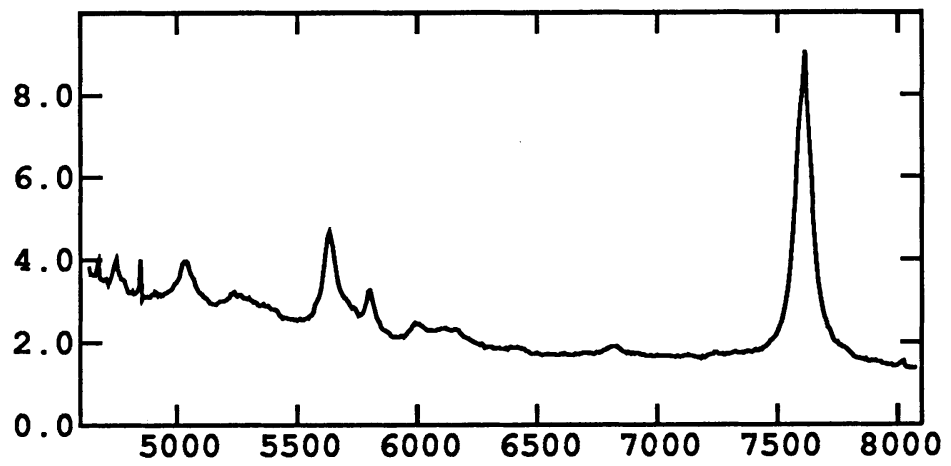

Figure 1. 1226+023 optical spectrum. Wavelength in Angstroms, vertical axis $\mathrm{F}_{\lambda}$ in $10^{-15}$ ergs s$^{-1} \mathrm{~cm}^{-2} \AA^{-1}$; resolution $\sim 14 \AA$.

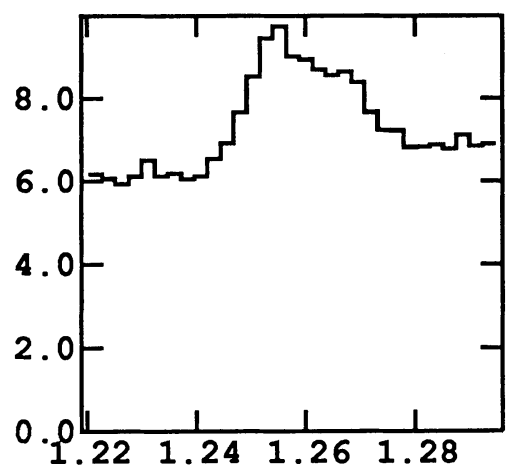

Figure 2. $1226+023 \mathrm{He} \mathrm{I} \lambda 10830$ and Pay. Wavelength in microns, vertical axis $\mathrm{F}_{\lambda}$ in $10^{-14} \mathrm{~W} \mathrm{~m}^{-2} \mu \mathrm{m}^{-1}$; resolution (1 pixel) $\sim 500$.

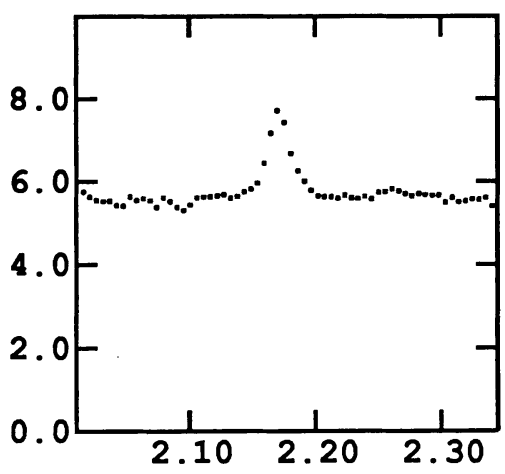

Figure 3. 1226+023 Pa . Wavelength and vertical axis as figure 2 ; resolution (FWHM) 200 . 